\title{
Beyond Growth Factors: Macrophage-Centric Strategies for Angiogenesis
}

\author{
Alexis L. Nolfi ${ }^{1,2} \cdot$ Marissa N. Behun ${ }^{1,2} \cdot$ Cecelia C. Yates ${ }^{2,3,4} \cdot$ Bryan N. Brown $^{1,2} \cdot$ Mangesh Kulkarni $^{1,2}$ (I)
}

Accepted: 12 October 2020 / Published online: 10 November 2020

(C) The Author(s) 2020

\begin{abstract}
Functional angiogenesis is a critical therapeutic goal in many pathological conditions. Logically, the use of pro-angiogenic growth factors has been the mainstay approach despite obvious limitations and modest success. Recently, macrophages have been identified as key regulators of the host response to implanted materials. Particularly, our understanding of dynamically plastic macrophage phenotypes, their interactions with biomaterials, and varied roles in different stages of angiogenic processes is evolving rapidly. In this review, we discuss changing perspectives on therapeutic angiogenesis, in relation to implantable materials and macrophage-centric strategies therein. Harnessing the different mechanisms through which the macrophagedriven host response is involved in angiogenesis has great potential for improving clinical outcome.
\end{abstract}

Keywords Biomaterial $\cdot$ Macrophage $\cdot$ Polarization $\cdot$ Angiogenesis $\cdot$ Growth factor $\cdot$ Ischemia

\section{Introduction}

The field of tissue engineering and regenerative medicine has long promised to provide transplantable materials, engineered constructs, and other approaches to heal damaged or dysfunctional tissues. Significant advancements have been made in the areas of biomaterials, drug delivery, and cellular engineering, enabling the development of advanced materials, cellular therapies, and tissue-like constructs. However, the long-term integration of any such material or construct will logically depend on its ability to interface with the existing vasculature, supplying cells, oxygen, and nutrients to the site of implantation [1-4].

This article is part of the Topical Collection on Wound Healing and Tissue Repair

Mangesh Kulkarni

mak441@pitt.edu

Alexis L. Nolfi

Aln24@pitt.edu

Marissa N. Behun

Mnb43@pitt.edu

Cecelia C. Yates

Cecelia.yates@pitt.edu

Bryan N. Brown

brownb@upmc.edu
Such vascularization is of critical importance, regardless of the strategy employed. Poor vascularization can lead to fibrotic tissue encapsulation, cellular death, tissue necrosis, and failure of the implant, among other complications [5-8]. Critically, vascularization of implantable materials is thought to be limited to several hundred micrometers $[1,9]$, significantly limiting the size and shape of engineered constructs and their ability to address significant tissue injury and disease.

Many approaches to inducing angiogenesis, the growth of blood vessels from existing vasculature, have been proposed. As angiogenesis is a key aspect of numerous biological processes spanning developmental biology, wound healing,

Department of Bioengineering, University of Pittsburgh, Suite 300, 450 Technology Drive, Pittsburgh, PA 15219, USA

2 McGowan Institute for Regenerative Medicine, University of Pittsburgh, Suite 300, 450 Technology Drive, Pittsburgh, PA 15219, USA

3 Department of Pathology, University of Pittsburgh, Suite 300, 450 Technology Drive, Pittsburgh, PA 15219, USA

4 Department of Health Promotion and Development, School of Nursing, University of Pittsburgh, Suite 300, 450 Technology Drive, Pittsburgh, PA 15219, USA 
organ transplantation, tumorigenesis, and numerous others, many have looked to the mechanistic studies of these processes as a guide for the development of approaches to the induction and maturation of blood vessels [2,3]. Largely, these efforts have focused upon the delivery of individual or multiple growth factors to recruit endothelial cells or their precursors and to direct their proliferation and differentiation. Others have investigated pre-vascularization of engineered constructs with endothelial cells to speed revascularization. However, as angiogenesis is a highly regulated process including temporal and spatial regulation of stimuli, cellular crosstalk, and matrix remodeling - dysregulation of which leads to abnormal or non-functional vascularizationfew current strategies can effectively control all aspects. For this reason, while some degree of pre-clinical success has been observed, few of these approaches have translated to clinically effective treatments.

Further complicating the design of engineered therapies, all materials and cellular constructs are subjected to the host immune response following implantation [10-15]. The nature of this response has been shown to be a key determinant of long-term integration and success $[12,13]$. Recently, significant attention has been paid to the phenotype of macrophages in the remodeling process. Macrophages, depending upon their activating stimuli, are now recognized to polarize into a spectrum of phenotypes with pro-inflammatory (M1) or "alternative" (M2) functions [16-19]. The alternatively activated M2 subset has generally been associated with improved outcomes following the implantation of biomaterials or engineered constructs $[12,13]$. However, the segmentation of macrophages into M1 and M2 subtypes is an oversimplification, and macrophages may express multiple, highly complex phenotypes which change throughout the response [20-22]. Thus, while the success of tissue engineering and regenerative medicine strategies depends critically upon the ability to orchestrate the highly complex and temporal process of angiogenesis, it also depends upon the ability to influence a similarly complex host immune response. Macrophages of multiple phenotypes have now been shown to be potent modulators of the angiogenic process [23-26], demonstrating that the host response and angiogenic response are inextricable from one another and must be considered in tandem to achieve improved outcomes.

The present review seeks to provide an overview of select approaches which have been used to promote angiogenesis, an overview of the role of macrophages in the vascularization of implantable materials and engineered constructs, and a brief review of clinical challenges in promoting angiogenesis. We suggest that approaches which consider the host response as a critical modulator of the angiogenic process have significant potential to result in improved outcomes.

\section{Changing Perspectives on Biomaterials Development for Promoting Angiogenesis}

Angiogenesis, or the formation of new blood vessels from preexisting vessels, is a tightly temporally and spatially regulated event in vivo requiring the presence of numerous cell types and biological signals and cues. A classical and simplistic view of sprouting angiogenesis relies upon hypoxic environments causing oxygen-sensing mechanisms to increase production of VEGF, leading to a chemical gradient, and inducing endothelial tip cell formation and growth of the newly sprouting vasculature towards the gradient. It follows, then, logically, that early strategies for inducing vascularization and promoting healing would make use of delivery of VEGF. In the field of bone tissue engineering, for example, vascularization is a critical component for successful regenerative outcomes and incorporation of scaffolds and grafts. Studies in the field have shown benefit from treatment with VEGF leading to better vascularization $[27,28]$.

Over the years, however, perspectives have changed, and we have come far in our understanding and the strategies that are employed. While VEGF therapy may recruit endothelial cells and drive early angiogenesis, vessel stabilization and maturation often necessitate other factors if the end goal is a well-vascularized scaffold. In addition, lack of control of VEGF concentration in a therapy (i.e., too much in an area) has been found to have detrimental effects on the wellorchestrated tip vs stalk cell selection and subsequent proliferation and movement necessary for sprouting angiogenesis [29]. Other key players include PDGF and FGF-2, as well as numerous other cytokines, chemokines, and ECM proteins [30]; therefore, it is understandable that modern strategies would attempt to incorporate or elicit other physiologically relevant components in their vascularization strategies. In addition, the ways in which we design biomaterials to have an angiogenic impact have changed. That is, previously overlooked components such as surface topography and modifications, scaffold cellular pre-seeding, and materials properties, among others, have all now been shown to influence vascularization potential.

One of these strategies, pre-vascularization of a scaffold, can have a profound effect on downstream vascularization and integration with existing vessels post-implantation. In general, engineered tissue constructs exceeding a thickness past which diffusion is not able to deliver nutrients and oxygen and remove wastes from all areas will benefit from a prevascularization strategy before implantation. For example, large bony defects are not always amenable to repair with an autograft remedy, and the use of an engineered alternative scaffold without pre-vascularization can result in issues with graft integration and poorly functioning tissue [31, 32]. Applications such as these, among others, would certainly benefit from a pre-vascularized scaffold. 
Unsurprisingly, pre-vascularized constructs have been found to have a quicker time to whole construct perfusion in vivo [33] when compared to growth factor delivery in the absence of pre-vascularization strategies [34]. Scaffold prevascularization can rely on a wide variety of biomaterials, cell types, and seeding strategies. Commonly an endothelial cell source is used with or without the presence of other cell types (e.g., fibroblasts), depending on the application. Endothelial cells are a critical component of new blood vessel formation and have historically been a prominent target for proangiogenic growth factor release strategies. Logically it would follow that incorporating exogenous endothelial cells into a strategy or scaffold could enhance angiogenesis, especially in implants that are difficult to perfuse [35]. A common source and type of endothelial cell are human umbilical vein endothelial cells (HUVECs), which, as a primary cell source, have less ethical considerations and are relatively easy to harvest and employ [36], in addition to having standardized and wellcharacterized assays and protocols for their use. However, despite utility in pre-clinical studies involving addition of HUVECs to scaffolds, their use may be limited by ability to be translated to the clinic, especially with respect to "personalized medicine."

Alternative sources of endothelial cells for scaffold seeding have become available and include endothelial cells derived from the dermal tissue [37, 38], endothelial colony forming cells from the peripheral blood [39], and endothelial cells derived from induced pluripotent stem cells (iPSCs) [40]. While there are challenges associated with each type, endothelial cells derived from iPSCs do have the obvious advantage of being able to provide an autologous supply of cells [41].

Just as important as determination of cellular components to include, biomaterial choice can influence the success of a pre-vascularization strategy. Biomaterials that mimic the tissues they will be implanted into or aim to replace (i.e., biomaterials that have appropriate mechanical, geometrical, and functional properties) will have a more promising chance at pre-vascularization and subsequent integration [42]. Natural biomaterials such as collagen, fibrin, gelatin, and decellularized extracellular matrix, among others, help to provide important biochemical and functional cues to cells during cell seeding [43], and upon implantation; however, synthetic materials, albeit oftentimes needing to be modified in order to be capable of pre-vascularization [44], show utility in terms of production consistency and lack of immunogenicity. For example, the synthetic polyether polyethylene glycol (PEG) has been successfully incorporated into numerous scaffold prevascularization strategies [45], as well as the synthetic ester biomaterials such as PLA, PGA, and PLGA.

In both the presence and absence of scaffold prevascularization strategies, the physical architecture, including topographical properties, of a biomaterial can influence and induce vascularization after implantation. Porosity is sometimes a tunable feature of a biomaterial that can be designed in a way to allow the nutrient transport and penetration of host cells necessary for a well-vascularized scaffold [46]. For example, in a study by Chiu et al., PEG hydrogel scaffolds became more quickly vascularized in culture when hydrogel pore sizes were increased, and gels with larger pore sizes had deeper vascularization in vivo [47]. Similarly, Bai et al. also showed that pores of a sufficient size were necessary for vascular penetration; however, pore interconnections within the scaffold were just as important [48].

Though delivery of a single pro-angiogenic molecule from a scaffold may not produce the most physiologically relevant vasculature network, there are still numerous applications and opportunities for strategies that employ such techniques with promising results $[49,50]$. However, a new understanding, coupled with enhanced delivery techniques (e.g., layer by layer deposition for drug delivery), has paved the way for strategies that aim to more closely mimic the temporal delivery of more than one angiogenic factor. In a recent study, Bai et al. showed an improved angiogenic response and vessel maturation in vitro using human umbilical vein endothelial cells when cells were incubated with a scaffold that quickly released VEGF and FGF-2 earlier on, followed by a slower delayed release of PDGF vs cells that were incubated with growth factors void of temporally specified release [51•]. Similarly, Tengood et al. showed that sequential delivery of bFGF followed by PDGF in a modified in vivo murine Matrigel plug assay with a hollow fiber delivery system produced better vascular integration versus non-sequential delivery of factors [52], the proposed mechanism of both being the initiation of endothelial sprouting with bFGF followed by vessel stabilization with PDGF at later time points. Interestingly, it is not just one specific sequence of factors, but many different combinations that have been tried that have shown promise with respect to enhanced vascularization [53-55].

As an extension to sequential delivery of pure factors known to have angiogenic effects, recent research has also focused on sequential delivery of cell-specific mixtures of factors. That is, by eliciting production of desired mixtures of angiogenic factors from appropriate cells in vivo, an ondemand and temporally relevant supply of factors is made possible, as opposed to delivering exogenous angiogenic factors, some possible risks of which include short half-life of delivered agent and/or inappropriate concentration or location of delivery $[56,57]$.

\section{Macrophages in Angiogenesis: Indirect Contributors or Key Determinants?}

Along with the obvious contribution of endothelial cells and smooth muscle cells, it is now evident that macrophages, 
fibroblasts, and pericytes play important roles in angiogenesis. Though fibroblasts have been associated with ECM production and scar formation, they also play an equally important role in angiogenesis [58]. Secretion of PDGF and TGF- $\beta$ by macrophages is responsible for influx of fibroblasts to the site, and then both macrophages and fibroblasts secrete proangiogenic factors such as VEGF and bFGF leading to formation of new blood vessels [59]. Pericytes, on the other hand, provide mechanical support for endothelial cells and play essential metabolic and signaling roles in angiogenesis in a tissue- and angiogenic-stage specific way [60]. The interaction of macrophages with endothelial cells is critical during sprouting angiogenesis [61]. Macrophages interact with endothelial cells through ICAM-1 and VCAM-1 and eventually lead to angiogenesis [62].

\section{Macrophage Activation State and Angiogenesis}

It is not unreasonable to draw an association between macrophages and biomaterials-induced angiogenesis, given their critical role in developmental angiogenesis [63]. In fact, it is increasingly evident that macrophages are not simply host immune responders to foreign biomaterials; rather macrophages adopt a range of responses leading to healing and regeneration by enhancing angiogenesis. The pro-angiogenic potential of macrophages blurs the boundaries of their traditionally defined M1 pro-inflammatory and M2 anti-inflammatory activation phenotypes. Since M2 macrophages are pro-regenerative and share many characteristics with tumor-associated macrophages, they have been traditionally associated with enhanced vascularization. A comparative study showed that M2, but not M1, macrophages promoted angiogenesis when transplanted into a subcutaneous pocket in vivo [64]. Moreover, studies using biomaterials loaded with macrophages polarized to the M2 phenotype showed increased angiogenesis over those with M1 macrophages [65]. On the contrary, M1 macrophages are known to secrete pro-angiogenic factors such as VEGF, bFGF, and IL-8, and some studies have attributed higher angiogenesis to M1 macrophages. These contrasting results speak to the overlapping and multiphasic nature of macrophage polarization in wound healing. The distinct aspects of angiogenesis affected by M1 or M2 macrophages have yet to be fully understood, and the results depend largely on the temporal timing of macrophage polarization. In fact, a study has shown that coordinated contributions from both M1 and M2 macrophages are required for angiogenesis and scaffold vascularization [25]. The authors showed distinct secretory profiles, especially pro- and anti-angiogenic factors based on the macrophage phenotype and complementary roles of different macrophage subsets, particularly in relation to implant angiogenesis [25]. Another study demonstrated, through extensive in vitro characterization of macrophage secretome and the use of an endothelial cell sprouting assay, that the presence of M1 macrophages induced cell migration, while the presence of M2 macrophages enhanced endothelial cell sprouting and induced endothelial network formation [66]. The relative importance of each macrophage phenotype may also depend on the type of wound and/or underlying disease. For example, chronic, particularly diabetic, wounds are characterized by chronic inflammation with overproduction of nitric oxide by macrophages and insufficient or non-functional angiogenesis. Therefore, a number of promising strategies have sought to elicit a tunable balance of arginine metabolism via nitric oxide synthase and its competitive pathway arginase by M1 and M2 macrophages, respectively.

\section{Mechanisms of Macrophage-Induced Vascularization of Biomaterials}

The pro-angiogenic and vascularization function of macrophages is executed through several mechanisms, summarized in Fig. 1. The main mechanism is thought to be via secretion of growth factors [67] and has been shown to be particularly relevant to macrophages associated with biomaterial implants [68]. However, other potential mechanisms such as their role as endothelial cell chaperones and/or transdifferentiation into support cells, as well as acting as key regulators of vessel sprouting, are equally important [69-71]. Direct or indirect facilitation of vessel anastomosis is another mechanism by which M2 macrophages have been suggested to enhance angiogenesis [69].

Another important mechanism by which macrophages may influence angiogenesis is via ECM remodeling. For example, macrophages not only secrete MMPs but also inhibitors of proteases and thus help to dictate controlled ECM degradation, paving the way for migrating cells [72]. Moreover, degradation of some ECM molecules such as hyaluronic acid or fibrin renders the molecules pro-angiogenic. In addition, enzymatic degradation of ECM components by macrophages leads to the release of several growth factors like bFGF, TGF-b, and GMCSF, which can then act on endothelial cells. ECM remodeling by macrophages alters the physical forces acting on ECs and thus influences their responsiveness to pro-angiogenic growth factors. Thus, ECM-based biomaterials can be tuned to take advantage of these mechanisms and enhance to the angiogenic response of these biomaterials. Another potential way macrophages influence angiogenesis is through transdifferentiation and vascular mimicry. It has been suggested that macrophages can start to express markers such as PECAM-1 and VECadherin which are endothelial cell markers [73].

\section{Biomaterials That Harness Macrophages to Drive Angiogenesis}

Early biomaterials research focused on shielding or "hiding" the material from the body and immune system, but our understanding has changed. Recent efforts to engage the 


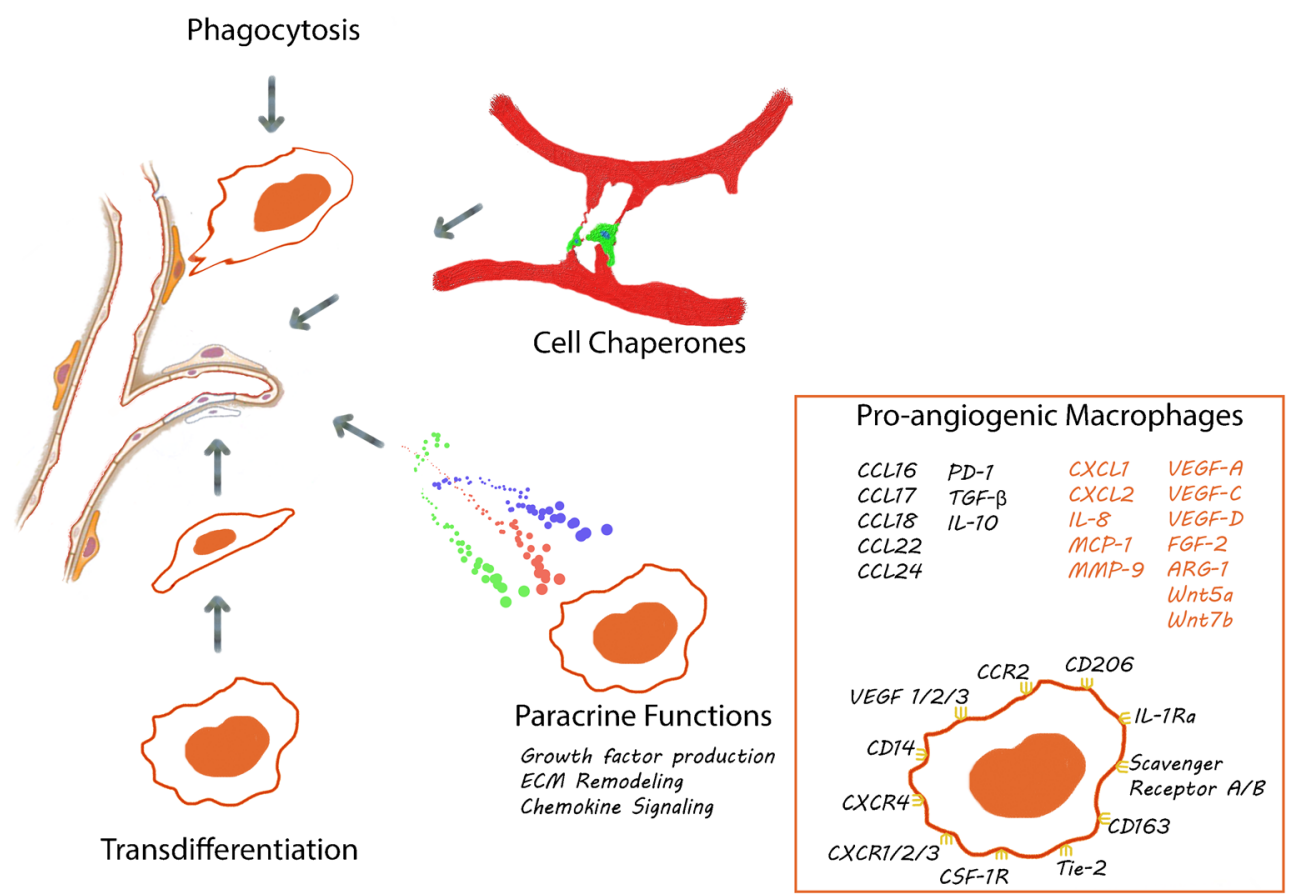

Fig. 1 Mechanisms through which macrophages are involved in promoting angiogenesis. A number of pro-angiogenic markers have been associated macrophages. In addition to production of proangiogenic growth factors, ECM remodeling enzymes, and chemokine signaling, macrophages are known to drive angiogenesis through many other mechanisms. Transdifferentiation to endothelial cells or vascular

immune system have shown promise, especially with respect to a greater understanding of the diversity and plasticity of macrophages. It is clear that macrophages play a critical role in implant integration, in part, by driving various aspects of angiogenesis. Therefore, the choice of biomaterial implants, surface modifications, and inclusion of biomolecules to attract macrophages to the site of implantation are among the strategies being used in the field to effectively harness the angiogenic potential of macrophages. A recent study demonstrated that simply altering the choice of synthetic biomaterial had a significant effect upon secretion of angiogenic molecules and macrophage phenotypes [74]. Another tissue engineering and regenerative medicine strategy in harnessing macrophages for angiogenesis is cell retention. Biomaterial encapsulation has been used as a strategy to effectively retain the cells at the site and has been shown to positively affect the angiogenic potential of macrophages in critical limb ischemia [75]. In bone regeneration, it has been demonstrated that macrophages positively influence the co-culture of endothelial cells and osteoblasts to form new microvessel-like structures [76].

Various aspects of biomaterial design such as chemical composition, size, shape, and mechanical properties can be tailored to modulate host immune response, and thereby, angiogenesis and downstream remodeling. Recent studies with peptide biomaterials demonstrated that the early immune response is determined by biomaterial design which can, in turn, mimicry has been reported through expression of endothelial markers. Phagocytosis and regulation of vessel sprouting have also been reported. Vessel anastomosis is an important aspect of functional angiogenic response, and macrophages have been shown to act as cell chaperones to facilitate the process. The schematic depicts macrophages in green, wrapping around the vessel during anastomosis

determine the downstream processes which elicit an angiogenic response [77•, 78]. Keeping the chemical composition but modifying the micro-architecture can also impart the biomaterial with certain properties conducive to a pro-angiogenic macrophage response. In fact, a recent study has shown that by engineering micro-channeled scaffolds, the macrophage response can be altered towards a more M2-like phenotype which drives an angiogenic response [79]. Another example of a biomaterial utilizing M2 macrophage polarization to enhance angiogenesis and tissue repair is the use of chitosan hydrogels for prolonged release of prostaglandin $E_{2}$ [80].

Macrophage M2 phenotypic transformation is particularly desirable in diabetic wounds. One such approach utilized Lnitroarginine-based polyester amide co-polymers to skew the balance towards M2 macrophages, leading to enhanced angiogenesis and healing [81]. In recent years, biomaterials in the form of nanoparticles are being used to induce healing. One report has shown promise by triggering the nanoclustering of mannose receptors on macrophages by modified nanoparticles, thereby inducing an anti-inflammatory response [82]. The same group further studied this mechanism with difficult to heal diabetic wounds and demonstrated that the differentiation of macrophages to the M2-like phenotype was associated with angiogenesis and eventually improved healing [83].

However, as discussed earlier, both M1 and M2 macrophages are essential for angiogenesis to progress properly 
for successful biomaterial integration. In this light, the biomaterial systems capable of modulating macrophage phenotype sequentially are deemed particularly rewarding for implant integration. The strategies and examples of sequential delivery systems for macrophage modulation have been reviewed recently [24]. This clearly demonstrates that materials which seek to avoid the host immune response may elicit poorer than desired remodeling outcomes due to poor vascularization.

\section{Clinical Strategies and Applications}

While there are many advanced cellular, growth factor, and biomaterials-based approaches to promoting angiogenesis and improved wound healing, few have yet to translate into clinical benefit. This is due to the often complex and/or expensive strategies employed in many of the above described pre-clinical approaches. It is also important to note that, while the present review has focused primarily upon pro-angiogenic strategies, primarily for wound healing, there are also a number of clinical conditions in which angiogenesis is pathogenic and undesirable. Currently, there are both pro- and antiangiogenic trials occurring in both pre-clinical and clinical settings. Some of the clinical trials focus on manipulating VEGF or its delivery to improve angiogenesis, while others use other resources such as hyperbaric oxygen treatments to stimulate angiogenesis. There are also anti-angiogenic clinical trials which focus on inhibiting ocular neovascularization.

\section{Peripheral Vascular Diseases}

The large majority of the angiogenic clinical trials being done currently are focused on finding a way to revascularize a diseased, ischemic heart. The FDA has recently approved two new laser treatments that are believed to help promote angiogenesis in this model. These lasers are being used as either a direct myocardial revascularization (DMR) or as a transmyocardial revascularization (TMR), with the overarching goal to promote new blood vessel formation in the heart [84, 85].

While laser treatments have been shown to provide an important angiogenic improvement, traditional treatments of direct administration of VEGF and FGF-2 have not shown the same improvements in patients. The VIVA clinical trial recently completed a phase I clinical trial where its goal was to deliver VEGF directly to the diseased heart tissue and to look for improved cardiac function $[84,86]$. This clinical trial focused on looking at a low and high dose of recombinant-VEGF (rhVEGF) administered in an intracoronary and intravenous way. Pre-clinical studies showed that the low dose of rhVEGF showed no statistical difference between the treatment and the placebo groups by day sixty. The higher dose groups were able to show an improvement in angina by the 120-day mark [86]. While these results were promising, they were shown to be only effective in the pre-clinical animal models and were not translatable to patients with progressive ischemia $[84,86]$. While the VIVA trials did not have a significant clinical impact, they did draw attention to the issues with delivering the VEGF-A protein. Direct delivery of VEGF-A intravenously was found to fail due to its short half-life. The protein degraded well before it was able to integrate into the ischemic tissue [86]. As most patients with ischemic heart conditions are not candidates for revascularization/CABG treatments, finding a way to deliver VEGF is important.

In the early 2000s, a new clinical trial, the GENESIS I trial, attempted to deliver naked plasmid DNA of VEGF directly into the myocardium using a NOGA catheter [87]. The results of this study were, however, not conclusive due to a significant observed placebo effect. A similar study was completed in Denmark that used proper blinded controls, but there were no significant differences between the treatment groups and the placebo groups [87].

A promising pre-clinical trial used an acellular tissue graft coated with small intestine submucosa, heparin, and VEGF that was able to capture blood monocytes, which ultimately resulted in recruitment of both endothelial cells and macrophages [88]. This study provides a hopeful new look at how, with the right coating, an acellular graft implanted in the artery of an ischemic animal model can engage circulating blood monocytes to vascularize the graft. If this model continues to show promise, it can be applied to various peripheral vascular diseases that currently use an allogenic or xenogeneic donor.

Another approach to further the utilization of macrophages in a pre-clinical angiogenic approach examined a Tie2expressing macrophage that could promote an angiogenic phenotype in endothelial cells [75]. The Tie-2 receptor is an angiopoietin receptor that, when activated by angiopoietins, can stimulate the formation of blood vessels. A recent study published by Ludwinski showed that by encapsulating murine bone marrow-derived macrophages expressing the Tie- 2 receptor and then seeding the cells with an alginate solution, an angiogenic profile was elicited in the cell population. By harnessing the angiogenic capabilities of the macrophages, angiogenesis was induced. As these pre-clinical trials move into the clinical phases, a more macrophage-focused approach may help to improve the clinical outcomes in peripheral vascular diseases.

Similar to the studies being done for ischemic heart, a phase I clinical trial is attempting to deliver naked plasmid DNA in a thromboangiitis obliterans (TAO) or Buerger's disease model. Plasmid DNA that encodes for VEGF (phVEGF ${ }_{165}$ ) was injected into the ischemic limbs of patients with Buerger's disease, with results consisting of both gene expression in blood samples and observations of unhealed ulcers before and after injection [89]. The outcomes of the study showed that patients who are treated with the plasmid 
before the onset of forefoot gangrene can have pain reduction, ulcer reduction, and increased distal blood flow. However, this study was not effective for any patient who had already developed forefoot gangrene $[89,90]$.

Another important clinical application of angiogenesis occurs in wound healing. Unlike the clinical trials for ischemic vascular disorders and Buerger's disease, most diabetic foot wound clinical trials are focused on utilizing hyperbaric oxygen treatments. Hyperbaric oxygen treatments (HBOTs) have been shown to promote angiogenic responses during wound healing [91, 92]. A systematic review released by Cochrane Database Systems showed that HBOT was able to significantly improve the healing of a diabetic foot after a year of treatment while also decreasing the need for amputation [92]. HBOTs have been shown to not only improve wound healing but have also shown an increase in function of key immune cells such as leukocytes and macrophages [91, 92], further suggesting that improvements in leukocyte and macrophage function may be beneficial in regard to a regenerative medicine approach for an affected limb.

\section{Ocular Diseases}

As a contrast to ischemic disease, angiogenesis in the eye can be detrimental, potentially leading to a disease state that could ultimately leave the patient blind. Such disease states include age-related macular degeneration, proliferative diabetic retinopathy, diabetic macular edema, and corneal neovascularization [93]. Anti-angiogenic trials have an important role in ocular therapies and treatments. The latest clinical application to combat ocular neovascularization uses a Fab fragment targeted against VEGF to help decrease neovascularization $[84,93]$. Additionally, treatments such as photodynamic therapies, ocular surface restoration, steroids, and VEGF inhibitors have been shown to successfully restore vascularity to normal levels. However, anti-angiogenic treatments are not just used for ocular applications but are also used widely in the treatment of cancer, theoretically depriving tumors of their ability to recruit blood vessels to supply nutrients, though to varying levels of success and generally in combination with chemotherapy or other treatments.

\section{Angiogenesis as a Diagnostic Tool}

Since abnormal angiogenesis can promote tumorigenesis and other abnormalities, there has been a shift in focus to use angiogenesis as a diagnostic tool. By understanding angiogenesis and measuring the microvessel density within a tissue biopsy, it is possible to determine if a patient has a predisposition for a specific cancer [94]. Additionally, urine tests evaluating the levels of bFGF in patient samples can be used as a biomarker for cancer predispositions. Understanding the mechanisms of angiogenesis has provided an important pathway for clinical testing to help prevent disease states $[84,85]$.

\section{Conclusion}

Angiogenesis is a critical factor in the host response to implantable materials, engineered constructs, and key to the treatment of many diseases and injuries. Current strategies for enhancing angiogenesis have been limited by challenges in translating approaches including growth factor delivery, advanced biomaterial design, and pre-vascularization. While increasing understanding of the angiogenic process and developments in materials science and cellular engineering continue to advance the ability to deliver successful angiogenic outcomes, any implantable material will be subject to a host inflammatory response. As macrophages are now recognized as key mediators of the angiogenic response, it is logical that tissue engineering and regenerative medicine approaches which consider angiogenesis and the host response in tandem may have improved outcomes in areas of significant clinical need.

\section{Compliance with Ethical Standards}

Conflict of Interest The authors have no potential conflicts of interest relevant to this article.

Animal Rights and Informed Consent This article does not contain any studies with human or animal subjects performed by any of the authors.

Open Access This article is licensed under a Creative Commons Attribution 4.0 International License, which permits use, sharing, adaptation, distribution and reproduction in any medium or format, as long as you give appropriate credit to the original author(s) and the source, provide a link to the Creative Commons licence, and indicate if changes were made. The images or other third party material in this article are included in the article's Creative Commons licence, unless indicated otherwise in a credit line to the material. If material is not included in the article's Creative Commons licence and your intended use is not permitted by statutory regulation or exceeds the permitted use, you will need to obtain permission directly from the copyright holder. To view a copy of this licence, visit http://creativecommons.org/licenses/by/4.0/.

\section{References}

Papers of particular interest, published recently, have been highlighted as:

- Of importance

1. Auger FA, Gibot L, Lacroix D. The pivotal role of vascularization in tissue engineering. Annu Rev Biomed Eng. 2013;15:177-200.

2. Mastrullo V, Cathery W, Velliou E, Madeddu P, Campagnolo P. Angiogenesis in tissue engineering: as nature intended? Front Bioeng Biotechnol. 2020;8:188. 
3. Rouwkema J, Khademhosseini A. Vascularization and angiogenesis in tissue engineering: beyond creating static networks. Trends Biotechnol. 2016;34:733-45.

4. Soker S, Machado M, Atala A. Systems for therapeutic angiogenesis in tissue engineering. World J Urol. 2000;18:10-8.

5. Jabbarzadeh E, Starnes T, Khan YM, Jiang T, Wirtel AJ, Deng M, et al. Induction of angiogenesis in tissue-engineered scaffolds designed for bone repair: a combined gene therapy-cell transplantation approach. Proc Natl Acad Sci U S A. 2008;105:11099-104.

6. Madden LR, Mortisen DJ, Sussman EM, Dupras SK, Fugate JA, Cuy JL, et al. Proangiogenic scaffolds as functional templates for cardiac tissue engineering. Proc Natl Acad Sci U S A. 2010;107: 15211-6.

7. Patel ZS, Mikos AG. Angiogenesis with biomaterial-based drugand cell-delivery systems. J Biomater Sci Polym Ed. 2004;15:70126.

8. Sharkawy AA, Klitzman B, Truskey GA, Reichert WM. Engineering the tissue which encapsulates subcutaneous implants. III. Effective tissue response times. J Biomed Mater Res. 1998;40: 598-605.

9. Colton CK. Implantable biohybrid artificial organs. Cell Transplant. 1995;4:415-36.

10. Anderson JM, Rodriguez A, Chang DT. Foreign body reaction to biomaterials. Semin Immunol. 2008;20:86-100.

11. Brown BN, Badylak SF. Expanded applications, shifting paradigms and an improved understanding of host-biomaterial interactions. Acta Biomater. 2013;9:4948-55.

12. Brown BN, Ratner BD, Goodman SB, Amar S, Badylak SF. Macrophage polarization: an opportunity for improved outcomes in biomaterials and regenerative medicine. Biomaterials. 2012;33: 3792-802.

13. Brown BN, Sicari BM, Badylak SF. Rethinking regenerative medicine: a macrophage-centered approach. Front Immunol. 2014;5: 510 .

14. Remes A, Williams DF. Immune response in biocompatibility. Biomaterials. 1992;13:731-43.

15. Zor F, Selek FN, Orlando G, Williams DF. Biocompatibility in regenerative nanomedicine. Nanomedicine (London). 2019;14: 2763-75.

16. Locati M, Curtale G, Mantovani A. Diversity, mechanisms, and significance of macrophage plasticity. Annu Rev Pathol. 2020;15: 123-47.

17. Mantovani A, Biswas SK, Galdiero MR, Sica A, Locati M. Macrophage plasticity and polarization in tissue repair and remodelling. J Pathol. 2013;229:176-85.

18. Mills CD. M1 and M2 macrophages: oracles of health and disease. Crit Rev Immunol. 2012;32:463-88.

19. Murray PJ, Wynn TA. Obstacles and opportunities for understanding macrophage polarization. J Leukoc Biol. 2011;89:557-63.

20. Hume DA. The many alternative faces of macrophage activation. Front Immunol. 2015;6:370.

21. Mosser DM, Edwards JP. Exploring the full spectrum of macrophage activation. Nat Rev Immunol. 2008;8:958-69.

22. Murray PJ, Allen JE, Biswas SK, Fisher EA, Gilroy DW, Goerdt S, et al. Macrophage activation and polarization: nomenclature and experimental guidelines. Immunity. 2014;41:14-20.

23. Corliss BA, Azimi MS, Munson JM, Peirce SM, Murfee WL. Macrophages: an inflammatory link between angiogenesis and lymphangiogenesis. Microcirculation. 2016;23:95-121.

24. O'Brien EM, Risser GE, Spiller KL. Sequential drug delivery to modulate macrophage behavior and enhance implant integration. Adv Drug Deliv Rev. 2019;149-150:85-94.

25. Spiller KL, Anfang RR, Spiller KJ, Ng J, Nakazawa KR, Daulton JW, et al. The role of macrophage phenotype in vascularization of tissue engineering scaffolds. Biomaterials. 2014;35:4477-88.
26. Vannella KM, Wynn TA. Mechanisms of organ injury and repair by macrophages. Annu Rev Physiol. 2017;79:593-617.

27. Geiger F, Lorenz H, Xu W, Szalay K, Kasten P, Claes L, et al. VEGF producing bone marrow stromal cells (BMSC) enhance vascularization and resorption of a natural coral bone substitute. Bone. 2007;41:516-22.

28. Li R, Stewart DJ, von Schroeder HP, Mackinnon ES, Schemitsch EH. Effect of cell-based VEGF gene therapy on healing of a segmental bone defect. J Orthop Res. 2009;27:8-14.

29. Bentley K, Gerhardt H, Bates PA. Agent-based simulation of notchmediated tip cell selection in angiogenic sprout initialisation. J Theor Biol. 2008;250:25-36.

30. Martino MM, et al. Extracellular matrix and growth factor engineering for controlled angiogenesis in regenerative medicine. Front Bioeng Biotechnol. 2015;3:45.

31. Garcia JR, Garcia AJ. Biomaterial-mediated strategies targeting vascularization for bone repair. Drug Deliv Transl Res. 2016;6: 77-95.

32. Kuss MA, et al. Prevascularization of 3D printed bone scaffolds by bioactive hydrogels and cell co-culture. J Biomed Mater Res B Appl Biomater. 2018;106:1788-98.

33. Chen X, Aledia AS, Popson SA, Him L, Hughes CCW, George SC. Rapid anastomosis of endothelial progenitor cell-derived vessels with host vasculature is promoted by a high density of cotransplanted fibroblasts. Tissue Eng A. 2010;16:585-94.

34. Hall H. Modified fibrin hydrogel matrices: both, 3D-scaffolds and local and controlled release systems to stimulate angiogenesis. Curr Pharm Des. 2007;13:3597-607.

35. Griffith CK, Miller C, Sainson RCA, Calvert JW, Jeon NL, Hughes $\mathrm{CCW}$, et al. Diffusion limits of an in vitro thick prevascularized tissue. Tissue Eng. 2005;11:257-66.

36. Baudin B, Bruneel A, Bosselut N, Vaubourdolle M. A protocol for isolation and culture of human umbilical vein endothelial cells. Nat Protoc. 2007;2:481-5.

37. Nor JE, et al. Engineering and characterization of functional human microvessels in immunodeficient mice. Lab Invest. 2001;81:45363.

38. Supp DM, Wilson-Landy K, Boyce ST. Human dermal microvascular endothelial cells form vascular analogs in cultured skin substitutes after grafting to athymic mice. FASEB J. 2002;16:797-804.

39. Lin Y, Weisdorf DJ, Solovey A, Hebbel RP. Origins of circulating endothelial cells and endothelial outgrowth from blood. J Clin Invest. 2000;105:71-7.

40. Taura D, Sone M, Homma K, Oyamada N, Takahashi K, Tamura $\mathrm{N}$, et al. Induction and isolation of vascular cells from human induced pluripotent stem cells-brief report. Arterioscler Thromb Vasc Biol. 2009;29:1100-3.

41. Palladino A, et al. Induced pluripotent stem cells as vasculature forming entities. J Clin Med. 2019;8:1782.

42. Tian L, George SC. Biomaterials to prevascularize engineered tissues. J Cardiovasc Transl Res. 2011;4:685-98.

43. O'Brien FJ. Biomaterials \& scaffolds for tissue engineering. Mater Today. 2011;14:88-95.

44. de Mel A, Jell G, Stevens MM, Seifalian AM. Biofunctionalization of biomaterials for accelerated in situ endothelialization: a review. Biomacromolecules. 2008;9:2969-79.

45. Phelps EA, Garcia AJ. Engineering more than a cell: vascularization strategies in tissue engineering. Curr Opin Biotechnol. 2010;21:704-9.

46. Brauker JH, Carr-Brendel VE, Martinson LA, Crudele J, Johnston WD, Johnson RC. Neovascularization of synthetic membranes directed by membrane microarchitecture. J Biomed Mater Res. 1995;29:1517-24.

47. Chiu YC, Cheng MH, Engel H, Kao SW, Larson JC, Gupta S, et al. The role of pore size on vascularization and tissue remodeling in PEG hydrogels. Biomaterials. 2011;32:6045-51. 
48. Bai F, Wang Z, Lu J, Liu J, Chen G, Lv R, et al. The correlation between the internal structure and vascularization of controllable porous bioceramic materials in vivo: a quantitative study. Tissue Eng A. 2010;16:3791-803.

49. Kawanabe T, Kawakami T, Yatomi Y, Shimada S, Soma Y. Sphingosine 1-phosphate accelerates wound healing in diabetic mice. J Dermatol Sci. 2007;48:53-60.

50. Tabata Y, Miyao M, Ozeki M, Ikada Y. Controlled release of vascular endothelial growth factor by use of collagen hydrogels. J Biomater Sci Polym Ed. 2000;11:915-30.

51. Bai Y, Bai L, Zhou J, Chen H, Zhang L. Sequential delivery of VEGF, FGF-2 and PDGF from the polymeric system enhance HUVECs angiogenesis in vitro and CAM angiogenesis. Cell Immunol. 2018;323:19-32 This study provides evidence that spatial and temporal release of multiple physiologically relevant angiogenic growth factors from a novel polymeric delivery system can enhance angiogenesis as opposed to delivery of solitary factors or factors void of temporal release.

52. Tengood JE, Ridenour R, Brodsky R, Russell AJ, Little SR. Sequential delivery of basic fibroblast growth factor and plateletderived growth factor for angiogenesis. Tissue Eng A. 2011;17: 1181-9.

53. Bayer EA, Fedorchak MV, Little SR. The influence of plateletderived growth factor and bone morphogenetic protein presentation on tubule organization by human umbilical vascular endothelial cells and human mesenchymal stem cells in coculture. Tissue Eng A. 2016;22:1296-304.

54. Bayer EA, et al. (*) Programmed platelet-derived growth factor-BB and bone morphogenetic protein-2 delivery from a hybrid calcium phosphate/alginate scaffold. Tissue Eng A. 2017;23:1382-93.

55. Tengood JE, Kovach KM, Vescovi PE, Russell AJ, Little SR. Sequential delivery of vascular endothelial growth factor and sphingosine 1-phosphate for angiogenesis. Biomaterials. 2010;31:780512 .

56. Crafts TD, Jensen AR, Blocher-Smith EC, Markel TA. Vascular endothelial growth factor: therapeutic possibilities and challenges for the treatment of ischemia. Cytokine. 2015;71:385-93.

57. Testa U, Pannitteri G, Condorelli GL. Vascular endothelial growth factors in cardiovascular medicine. J Cardiovasc Med (Hagerstown). 2008;9:1190-221.

58. Weihrauch D, Arras M, Zimmermann R, Schaper J. Importance of monocytes/macrophages and fibroblasts for healing of micronecroses in porcine myocardium. Mol Cell Biochem. 1995;147:13-9.

59. Olczyk P, Mencner L, Komosinska-Vassev K. The role of the extracellular matrix components in cutaneous wound healing. Biomed Res Int. 2014;2014:747584.

60. Bergers G, Song S. The role of pericytes in blood-vessel formation and maintenance. Neuro-Oncology. 2005;7:452-64.

61. Outtz HH, Tattersall IW, Kofler NM, Steinbach N, Kitajewski J. Notch1 controls macrophage recruitment and notch signaling is activated at sites of endothelial cell anastomosis during retinal angiogenesis in mice. Blood. 2011;118:3436-9.

62. Kalucka J, Bierhansl L, Wielockx B, Carmeliet P, Eelen G. Interaction of endothelial cells with macrophages-linking molecular and metabolic signaling. Pflugers Arch. 2017;469:473-83.

63. Arnold T, Betsholtz C. Correction: the importance of microglia in the development of the vasculature in the central nervous system. Vasc Cell. 2013;5:12.

64. Jetten N, Verbruggen S, Gijbels MJ, Post MJ, de Winther MPJ, Donners MMPC. Anti-inflammatory M2, but not proinflammatory M1 macrophages promote angiogenesis in vivo. Angiogenesis. 2014;17:109-18.

65. Wang M, Yu Y, Dai K, Ma Z, Liu Y, Wang J, et al. Improved osteogenesis and angiogenesis of magnesium-doped calcium phosphate cement via macrophage immunomodulation. Biomater Sci. 2016;4:1574-83.

66. Beyer S, Koch M, Lee YH, Jung F, Blocki A. An in vitro model of angiogenesis during wound healing provides insights into the complex role of cells and factors in the inflammatory and proliferation phase. Int J Mol Sci. 2018;19:19.

67. Koh TJ, DiPietro LA. Inflammation and wound healing: the role of the macrophage. Expert Rev Mol Med. 2011;13:e23.

68. Garg K, Sell SA, Madurantakam P, Bowlin GL. Angiogenic potential of human macrophages on electrospun bioresorbable vascular grafts. Biomed Mater. 2009;4:031001.

69. Fantin A, Vieira JM, Gestri G, Denti L, Schwarz Q, Prykhozhij S, et al. Tissue macrophages act as cellular chaperones for vascular anastomosis downstream of VEGF-mediated endothelial tip cell induction. Blood. 2010;116:829-40.

70. Hsu CW, et al. Improved angiogenesis in response to localized delivery of macrophage-recruiting molecules. PLoS One. 2015;10:e131643.

71. Yamamoto S, Muramatsu M, Azuma E, Ikutani M, Nagai Y, Sagara $\mathrm{H}$, et al. A subset of cerebrovascular pericytes originates from mature macrophages in the very early phase of vascular development in CNS. Sci Rep. 2017;7:3855.

72. Butoi E, Gan AM, Tucureanu MM, Stan D, Macarie RD, Constantinescu C, et al. Cross-talk between macrophages and smooth muscle cells impairs collagen and metalloprotease synthesis and promotes angiogenesis. Biochim Biophys Acta. 2016;1863: 1568-78.

73. Barnett FH, Rosenfeld M, Wood M, Kiosses WB, Usui Y, Marchetti V, et al. Macrophages form functional vascular mimicry channels in vivo. Sci Rep. 2016;6:36659.

74. Dagtekin G, Schiffer R, Klein B, Jahnen-Dechent W, ZwadloKlarwasser G. Modulation of angiogenic functions in human macrophages by biomaterials. Biomaterials. 2003;24:3395-401.

75. Ludwinski FE, Patel AS, Damodaran G, Cho J, Furmston J, Xu Q, et al. Encapsulation of macrophages enhances their retention and angiogenic potential. NPJ Regen Med. 2019;4:6.

76. Dohle E, et al. Macrophage-mediated angiogenic activation of outgrowth endothelial cells in co-culture with primary osteoblasts. Eur Cell Mater. 2014:27:149-64; discussion 164-145.

77. Lopez-Silva TL, et al. Chemical functionality of multidomain peptide hydrogels governs early host immune response. Biomaterials. 2020;231:119667 This study demonstrated the effect of chemical funcitonality displayed on the surface of multidomain peptide biomaterials on early host response and angiogenesis.

78. Moore AN, Lopez Silva TL, Carrejo NC, Origel Marmolejo CA, Li IC, Hartgerink JD. Nanofibrous peptide hydrogel elicits angiogenesis and neurogenesis without drugs, proteins, or cells. Biomaterials. 2018;161:154-63.

79. Won JE, Lee YS, Park JH, Lee JH, Shin YS, Kim CH, et al. Hierarchical microchanneled scaffolds modulate multiple tissueregenerative processes of immune-responses, angiogenesis, and stem cell homing. Biomaterials. 2020;227:119548.

80. Zhang S, Liu Y, Zhang X, Zhu D, Qi X, Cao X, et al. Prostaglandin E2 hydrogel improves cutaneous wound healing via M2 macrophages polarization. Theranostics. 2018;8:5348-61.

81. He M, Sun L, Fu X, McDonough SP, Chu CC. Biodegradable amino acid-based poly(ester amine) with tunable immunomodulating properties and their in vitro and in vivo wound healing studies in diabetic rats' wounds. Acta Biomater. 2019;84:114-32.

82. Gan J, Dou Y, Li Y, Wang Z, Wang L, Liu S, et al. Producing antiinflammatory macrophages by nanoparticle-triggered clustering of mannose receptors. Biomaterials. 2018;178:95-108.

83. Gan J, Liu C, Li H, Wang S, Wang Z, Kang Z, et al. Accelerated wound healing in diabetes by reprogramming the macrophages with particle-induced clustering of the mannose receptors. Biomaterials. 2019;219:119340. 
84. Giacca M, Zacchigna S. VEGF gene therapy: therapeutic angiogenesis in the clinic and beyond. Gene Ther. 2012;19:622-9.

85. Lee SU, Wykrzykowska JJ, Laham RJ. Angiogenesis: bench to bedside, have we learned anything? Toxicol Pathol. 2006;34:3-10.

86. Henry TD, Annex BH, McKendall G, Azrin MA, Lopez JJ, Giordano FJ, et al. The VIVA trial: vascular endothelial growth factor in ischemia for vascular angiogenesis. Circulation. 2003;107:1359-65.

87. Post MJ, Laham RJ, Kuntz RE, Novicki D, Simons M. The effect of intracoronary fibroblast growth factor-2 on restenosis after primary angioplasty or stent placement in a pig model of atherosclerosis. Clin Cardiol. 2002;25:271-8.

88. Smith RJ Jr, Nasiri B, Kann J, Yergeau D, Bard JE, Swartz DD, et al. Endothelialization of arterial vascular grafts by circulating monocytes. Nat Commun. 2020;11:1622.

89. Isner JM, et al. Treatment of thromboangiitis obliterans (Buerger's disease) by intramuscular gene transfer of vascular endothelial growth factor: preliminary clinical results. $J$ Vasc Surg. 1998;28: 964-73; discussion 973-965.
90. Brissova M, Shostak A, Shiota M, Wiebe PO, Poffenberger G, Kantz J, et al. Pancreatic islet production of vascular endothelial growth factor-a is essential for islet vascularization, revascularization, and function. Diabetes. 2006;55:2974-85.

91. Bhutani S, Vishwanath G. Hyperbaric oxygen and wound healing. Indian J Plast Surg. 2012;45:316-24.

92. C. J. Buckley, J. S. Cooper, "Hyperbaric, angiogenesis" in StatPearls. (Treasure Island (FL), 2020).

93. Maddula S, Davis DK, Maddula S, Burrow MK, Ambati BK. Horizons in therapy for corneal angiogenesis. Ophthalmology. 2011;118:591-9.

94. Brown AP, Citrin DE, Camphausen KA. Clinical biomarkers of angiogenesis inhibition. Cancer Metastasis Rev. 2008;27:415-34.

Publisher's Note Springer Nature remains neutral with regard to jurisdictional claims in published maps and institutional affiliations. 